Jpn. J. Med. Sci. Biol., 50, 241 - 250, 1997.

\title{
INCREASING CIPROFLOXACIN RESISTANCE AMONG PREVALENT URINARY TRACT BACTERIAL ISOLATES IN BANGLADESH
}

\author{
Jabed IQBAL, Mizanur RAHMAN, M. Shahidul KABIR \\ and Mushfiqur RAHMAN1
}

Department of Microbiology, and 1Department of Immunology, National Diagnostic Network, Diabetic Association of Bangladesh, 69-M Green Road, Panthopath, Dhaka-1205, Bangladesh

(Received October 23, 1997. Accepted June 1, 1998)

SUMMARY: Ciprofloxacin was evaluated along with other commonly used antibiotics against a total of 425 clinical isolates obtained from urine samples. Samples were collected from outdoor patients from different parts of Dhaka city. Susceptibility tests were done by the standardized disk diffusion method. Among the tested drugs, the percent susceptible rates observed were: ciprofloxacin $(74 \%)$, ampicillin (29\%), cephalexin (54\%) and trimethoprim-sulfamethoxazole (43\%) tested against all organisms; gentamicin (73\%) tested against gram-negative or -ganisms and erythromycin (72\%) tested against gram-positive organisms. Ciprofloxacin showed better activity against gram-negative isolates $(80 \%)$ compared to the other antibiotics. However, strains highly resistant to ciprofloxacin were detected among commonly isolated gram-negative urinary pathogens: Escherichia coli (18\%), Klebsiella species (19\%) and Pseudomonas species (30\%). Overall susceptibility rate for gram-positive cocci was significantly low for all the antibiotics tested including ciprofloxacin (62\%). Minimal inhibitory concentration (MIC) of ciprofloxacin was measured for all resistant and susceptible urinary tract infection (UTI) isolates. This study indicates emerging ciprofloxacin resistance among most UTI bacterial pathogens. Increasing resistance against ciprofloxacin demands coordinated monitoring of its activity, and rationale use of the antibiotic in UTI. 


\section{INTRODUCTION}

Urinary tract infections are among the most common clinical problems in medical practice. Enterobacteriaceae are responsible for most nonnosocomial, uncomplicated UTI, $E$. coli being the most common. The antibiotics that have been traditionally used against both complicated and uncomplicated UTI include ampicillin, trimethoprim-sulfamethoxazole, cephalexin, gentamicin, amoxicillin, nalidixic acid and nitrofurantoin. However, resistance to most of these antibiotics are widespread and quite high in different geographic regions (1). Gentamicin, because of its considerable toxicity, has restricted use in clinical practice. Specific urinary antiseptics like nalidixic acid or nitrofurantoin are limited to lower UTI, while resistant mutants develop quite rapidly in susceptible bacterial populations, in case of nalidixic acid. Use of these antimicrobial agents has reduced substantially along with the dramatic rise in fluoroquinolone use, particularly ciprofloxacin, in UTI.

The fluoroquinolones are relatively new broad-spectrum antibiotics which replaced nalidixic acid and other quinolones in the late 1980's. The members of this newer group include ciprofloxacin, lomefloxacin, norfloxacin, ofloxacin, pefloxacin, etc. Ciprofloxacin has been the most widely and frequently prescribed antimicrobial agent against both gram-negative and gram-positive aerobic bacteria $(2,3)$. The use of this broad-spectrum antibiotic has significantly altered the antimicrobial therapy in UTI and other systemic infections. Ciprofloxacin, with its concentrating ability in urine and high renal clearance, has become highly effective in the treatment of UTI $(4,21)$. However, ofloxacin has also higher bioavailability than many other fluoroquinolones and is also a potent antimicrobial agent in UTI treatment $(5,6)$. In Bangladesh, ciprofloxacin has been one of the most common broad-spectrum antibiotic used in UTI and also in other infections.

Mechanism of action of the fluroquinolones is primarily inhibition of DNA gyrase A which is required for bacterial DNA replication. With the widespread use of fluroquinolones, there have been reports of evolving bacterial resistance to ciprofloxacin. This has been observed among several bacterial species in different geographical areas of the world (7-10). The mechanisms by which these bacterial populations have become resistant to ciprofloxacin so quickly are multiple: mutation in the DNA gyrase A gene or protein, permeability changes due to mutation in OMP-F and efflux mechanisms (8). Although, ofloxacin belongs to the same fluroquinolone family of drugs, it has been less affected by these resistance 
mechanisms and has a lower resistance rate among staphylococcal isolates $(11,12)$. There has not been any'well-defined and focused study in our country regarding ciprofloxacin resistance among UTI or other clinical isolates and there are indications that ciprofloxacin-resistant microbial agents are increasing in Bangladesh. In our laboratory, isolation of ciprofloxacin-resistant bacterial strains from the urinary tract and development of ciprofloxacin resistance in bacterial pathogens associated with fluoroquinolone treatment failures have been noted. Therefore, to assess the current degree of ciprofloxacin activity and resistance, an in vitro clinical isolate study was conducted in the clinical microbiology laboratory.

\section{MATERIALS AND METHODS}

A total of 425 consecutive UTI isolates, obtained during the period of September 1996 to May 1997, were tested for antibiotic susceptibility in the microbiology laboratory. Among the isolates studied were E. coli (179), Klebsiella species (31), Acinetobacter species (28), Pseudomonas species (43), Proteus species (17), $S$. aureus (51), coagulase-negative staphylococci (44) and enterococci (32). Ciprofloxacin $(5 \mu \mathrm{g})$, ampicillin $(10 \mu \mathrm{g})$, cephalexin $(30 \mu \mathrm{g})$, erythromycin $(15 \mu \mathrm{g})$, gentamicin $(10 \mu \mathrm{g})$, and trimethoprim-sulfamethoxazole $(25 \mu \mathrm{g})$ antibiotic disks (Unipath Ltd., UK) were tested against the isolates. Ampicillin, ciprofloxacin, cephalexin, and trimethoprim-sulfamethoxazole were tested against both grampositive and -negative bacteria. Erythromycin was tested against gram-positive bacteria only, while gentamicin was tested against gram-negative bacteria. Susceptibility studies were performed according to the standardized disc-diffusion agar technique following NCCLS (1993a) standards (13). Zone diameters measured on Mueller-Hinton agar were interpreted as susceptible, moderately susceptible and resistant. MIC tests were performed according to NCCLS (1993b) standards with serial double dilutions of the antibiotic in Mueller-Hinton broth with inocula from fresh cultures in exponential growth phase (14).

The presence of infection was correlated with either microscopic evidence of infection in urine (pus cells) or clinical features of UTI or both. Patients with clinical features of uncomplicated UTI, e.g., cystitis or pyelonephritis were selected for this study. A cut-off value of $10^{4} \mathrm{CFU} / \mathrm{ml}$ was selected for further processing of the isolate. The $S$. aureus isolates were processed irrespective of number of CFU/ml. Only fast-growing and most prevalent organisms were tested against the antibiotics. Escherichia coli ATCC 25922 and Staphylococcus aureus ATCC 22923 were used as control strains. 


\section{RESULTS}

All samples included in the study were obtained from community patients from different regions in and around Dhaka city. Table I shows the percent susceptible rate of UTI isolates for ciprofloxacin and the other antibiotics. Only commonly isolated and fast-growing strains were included in the study. Results from this table show high resistance among most organisms against ciprofloxacin except Acinetobacter and Proteus species, which showed susceptibility of $84 \%$ and $88 \%$, respectively. The $E$. coli isolates ( $81 \%$ ) were less susceptible to ciprofloxacin than the above-mentioned two isolates, followed by Klebsiella (77\%) and Pseudomonas (70\%) species. The susceptibility is even lower in gram-positive organisms; all common antibiotics including ciprofloxacin exhibit modest activity against the isolates, susceptibility ranging from $38 \%$ to $72 \%$. However, cephalexin and erythromycin show a higher efficacy than ciprofloxacin against gram-positive isolates, the percent susceptible rate being 65,72 and $62 \%$, respectively, for the three antibiotics (Table I).

A total of 94 resistant isolates were processed for MIC testing, along with the sensitive strains. All of the resistant strains were confirmed as being refractory to ciprofloxacin inhibition (MIC $4 \mu \mathrm{g} / \mathrm{ml}$ ). E. coli species were most susceptible to ciprofloxacin, followed by Proteus species, while Pseudomonas species showed higher MICs $(0.25-1.0 \mu \mathrm{g} / \mathrm{ml})$ (Table II). Compared to other resistant gram-negative isolates, Pseudomonas species showed a higher level of ciprofloxacin resistance. Compared with the Gram-negative isolates, the results for gram-positive isolates were quite different. The MICs were considerably higher and among the resistant gram-positive isolates, enterococci showed a very high level of resistance $(16-32 \mu \mathrm{g} / \mathrm{ml})$.

The incidence of multi-drug resistance (MDR) among UTI isolates was also surveyed in the study (Table III). Incidence of multiple-drug resistance studied was higher in almost all ciprofloxacin-resistant groups. Gram-negative or -positive pathogens that were resistant to at least four drugs, irrespective of ciprofloxacin susceptibility, were considered multi-drug resistant. Sensitivity to other drugs were also tested, e.g., tetracycline and amoxycillin (not shown here). Significantly higher incidence of MDR was seen in ciprofloxacin-resistant isolates and the incidence was highest in Proteus species (100\%), while it was lowest for enterococci (13\%). Pseudomonas species showed highest incidence of MDR among the ciprofloxacin-sensitive group of isolates (24\%). 


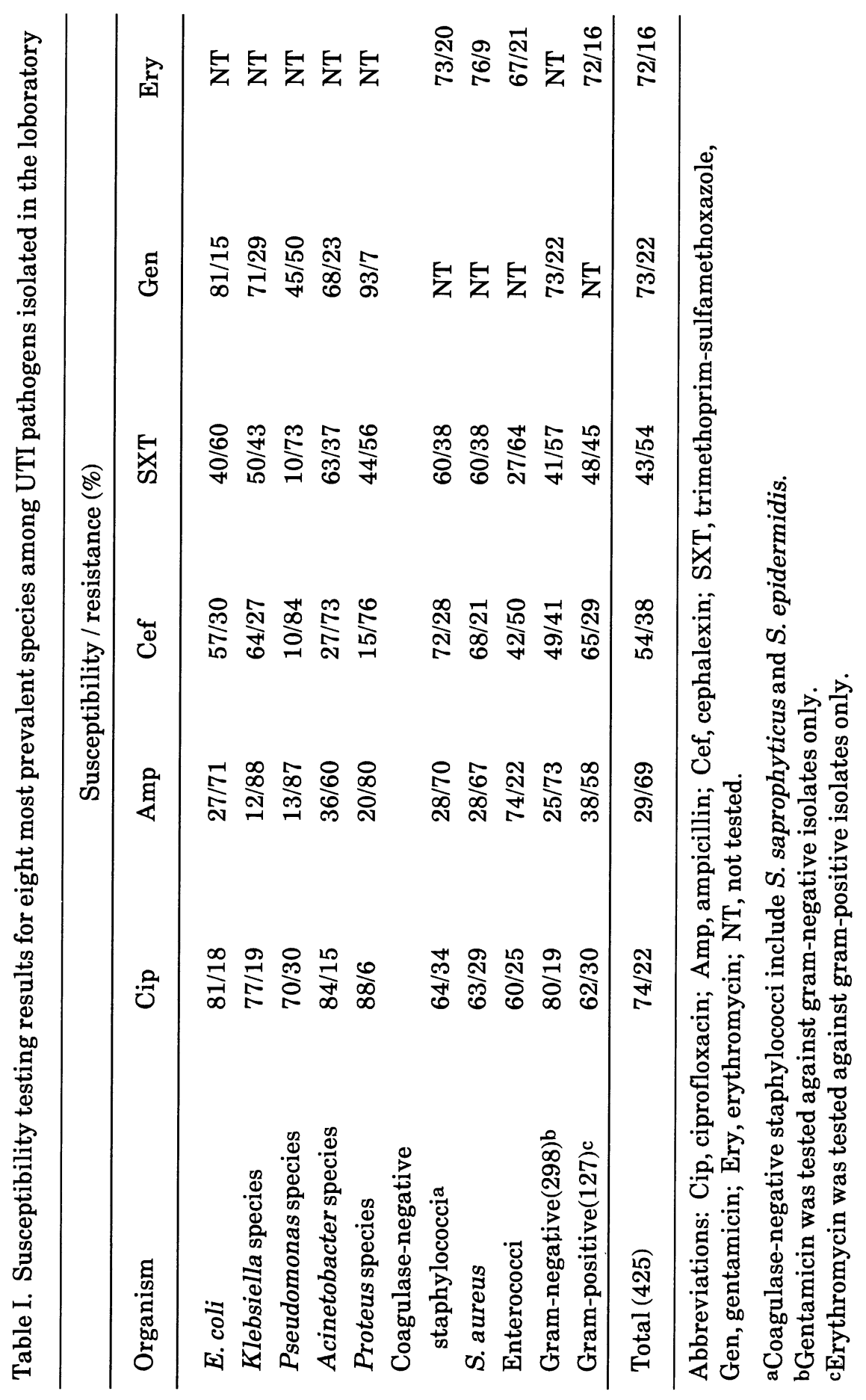


Table II. In vitro activity of ciprofloxacin against susceptible and resistant UTI isolates

\begin{tabular}{lll}
\hline & Minimal inhibitory concentration $(\mathrm{MIC}) \mathrm{a}(\mu \mathrm{g} / \mathrm{ml})$ \\
\hline Organism tested & Susceptible isolates & Resistant isolates \\
E. coli & $0.008-0.06$ & $4-32$ \\
Klebsiella species & $0.03-0.05$ & $4-32$ \\
Pseudomonas species & $0.25-1.0$ & $8-32$ \\
Acinetobacter species & $0.03-0.05$ & $4-8$ \\
Proteus species & $0.015-0.03$ & 4 \\
S. aureus & $0.25-0.5$ & $8-32$ \\
Coagulase-negative & & \\
$\quad$ staphylococcib & $0.125-0.5$ & $8-16$ \\
Enterococci & 2 & $16-32$ \\
\hline
\end{tabular}

aMIC $>(4 \mu \mathrm{g} / \mathrm{ml})$ was considered as resistant to ciprofloxacin.

bCoagulase-negative staphylococci include $S$. epidermis \& S. saprophyticus

Table III. Incidence of multi-drug resistance (MDR)a in ciprofloxacin-resistant (CR) and ciprofloxacin-sensitive (CS) isolates among UTI bacterial pathogens

\begin{tabular}{lcc}
\hline Organism tested & MDR among CR isolates (\%) & MDR among CS isolates (\%) \\
E. coli & 44 & 8 \\
Klebsiella species & 50 & 8 \\
Acinetobacter species & 50 & 13 \\
Proteus species & 38 & 24 \\
Pseudomonas species & 100 & 20 \\
Coagulase-negative & 62 & 3 \\
$\quad$ staphylococcib & 53 & 3 \\
S. aureus & 13 & 0 \\
Enterococci & & \\
\hline
\end{tabular}

aCross-resistance to ampicillin, trimethoprim-sulfamethoxazole, cephalexin and gentamicin (for gram-negative isolates) or erythromycin (for grampositive isolates) along with other antibiotics, irrespective of ciprofloxacin susceptibility. bIncludes $S$. epidermidis and $S$. saprophyticus. 


\section{DISCUSSION}

The primary objective of this study was to study the ciprofloxacin-resistance among UTI bacterial pathogens. Although ciprofloxacin has been introduced in Bangladesh in relatively recent years, this study indicates a fairly high resistance against this broad-spectrum antibiotic. Reports from different microbiology laboratories have also indicated increasing resistance among UTI isolates against ciprofloxacin (22). Possibly, injudicious use of the board-spectrum antibiotic by physicians and access of patients to the drug without proper prescription, are significant causes of this resistance in our country. Although, studies conducted on UTI isolates in North America have shown a higher activity of fluoroquinolones against most bacterial isolates, ciprofloxacin resistance appears to be higher among $E$. coli and other common UTI isolates in Bangladesh $(15,16)$. Reports regarding ciprofloxacin resistance in Bangladesh show susceptibility of E. coli ranging from $60 \%$ in diabetic patients to $96 \%$ in nondiabetic patients (18). Another study conducted on a pediatric population of 50 hospitalized patients, with UTI, showed $100 \%$ susceptibility of $E$. coli against ciprofloxacin (17). In contrast, another group in Pakistan has shown $81 \%$ susceptibility aganisnt ciprofloxacin in a pediatric population (19). Our study comprised of urban adult patients showed significant ciprofloxacin resistance among $E$. coli and most UTI bacterial pathogens. However, further study is required to establish any difference in susceptibility pattern of urinary pathogens against fluoroquinolones in the pediatric population.

The results obtained in this study revealed several other antimicrobial susceptibility pattern: (a) ampicillin is the least effective antimicrobial agents studied here, with high resistance against the antibiotic being prevalent among all UTI pathogens including enterococci (b) trimethoprim-sulfamethoxazole (SXT) is becoming increasingly ineffective against most gram-negative bacilli and grampositive cocci (c) cephalexin and erythromycin show good activity against most Gram-positive cocci and appear to be more effective than ciprofloxacin. These observations regarding ampicillin, cephalexin or SXT efficacy are in concurrence with data from previous studies in our country (1). On the other hand, gentamicin, appears to be more effective than ciprofloxacin against $E$. coli, the resistance rate being lower (15\% vs. $18 \%$ ). Due to its parenteral form and higher toxicity, gentamicin use has been somewhat limited in Bangladesh. Apparently, this may have restricted development of gentamicin resistance among $E$. coli isolates. 
Most strains of commonly isolated gram-negative bacteria showed good susceptibility to ciprofloxacin and MIC's for these organisms were well below 0.5 $\mathrm{mg} / \mathrm{ml}$, with the exception of Pseudomonas species. On the other hand, ciprofloxacin and all other antibiotics included in the study showed moderate activities against gram positive isolates. Ciprofloxacin MIC was quite high among most resistant gram-positive isolates indicating a high level of fluroquinolone resistance among this group of UTI isolates. The incidence of multi-drug resistance was also high in case of ciprofloxacin-resistant $S$. aureus and coagulase-negative staphylococci. This may be explained by the increasing percentage of methicillin (oxacillin) resistance, about 30\%, among staphylococcal isolates (unpublished data). Studies conducted in other regions indicate that cross-resistance occurs between oxacillin and fluroquinolones as a group, as well as other antibiotics (15).

The high incidence of multi-drug resistance among ciprofloxacin-resistant strains, as observed in our study, is significant in terms of infection control, particularly in hospitalized patients. This resistance to structurally unrelated antimicrobial agents indicate either mutations decreasing drug permeation or high prevalence of plasmid-mediated drug resistance, e.g., against aminoglycosides or cephalosporins $(15,20)$. Similar surveillance studies conducted on both hospitalized and outdoor patients also indicate high incidence of multi-drug resistance. $\mathrm{F}$. Jinnah and his co-workers have shown in their study that $34 / 65$, i.e., $52 \%$ E. coli urinary isolates were resistant to four or more antibiotics, the incidence being $86 \%$ in hospitalized diabetic patients and $42 \%$ in non-diabetic outdoor patients (18). Our study has shown similar data in case of $E$. coli isolates of outdoor patients (Table I). Attention should also be paid to emergence of oxacillin-resistant $S$. aureus strains which are more resistant not only to fluroquinolones but also to cephalosporins and carbapenems.

Most studies conducted abroad have indicated superiority of ofloxacin over ciprofloxacin, in terms of its activity against clinical isolates $(15,16)$. We are now conducting a surveillance study comparing the activity of ciprofloxacin with other fluroquinolones and preliminary data indicate similar trends. The use of ofloxacin is almost negligible in our region and it may be considered as an alternative to ciprofloxacin, since there is an indication of emerging resistance against the latter. We recommend that national level antimicrobial surveillance studies be carried out to monitor emerging antimicrobial resistance to help clinicians determine better empiric, prophylactic and specific antibiotic therapy to obtain best clinical results. Use of ciprofloxacin should be more rationale and indiscriminate use of the drug should be discouraged. Other members of this group, e.g., 
ofloxacin, may be considered and controlled use of the drug as an altrenative to ciprofloxacin may be advocated.

\section{ACKNOWLEDGEMENTS}

We thank Dr. A. L. Mallik, Coordinator, National Diagnostic Network, for his cooperation and support. We would like to express our gratitude to Diabetic Association of Bangladesh for providing finacial support for this project. We also thank all our colleagues at NDN for their help in continuing this study.

\section{REFERENCES}

1. Akhtar, N., Mowlah, G., Shufi, G. B., Mowlah, A. and Hoque, R. (1995): Resistance of urinary tract bacterial pathogens. Bangladesh J. Microbiol., 12, 97-100.

2. Monk, J. P. and Campoli-Richards, D. M. (1987): Ofloxacin: a review of its antibacterial activity, pharmacokinetic properties and therapeutic use. Drugs, 33, 346-391.

3. Campoli-Richards, D. M., Monk, J. P., Price, A., Benfield, P. and Todd, P. A. (1988): Ciprofloxacin: a review of its antibacterial activity, pharmacokinetic properties and therapeutic use. Drugs, 35, 373-447.

4. Piddock, L. J. V. and Wise, R. (1989): Mechanisms of reistance to quinolones and clinical perspectives. J. Antimicrob. Chemother., 23, 475-483.

5. Wolfson, J. S. and Hooper, D. C. (1989): Comparative pharmacokinetics of ofloxacin and ciprofloxacin. Am. J. Med., 87 (Suppl. C), 6-31.

6. Todd, P. A. and Faulds, D. (1991): Ofloxacin, a reappraisal of its antimicrobial activity, pharmacology and therapeutic use. Drugs, 42S, 825-876.

7. Schaefler, S. (1988): Methicillin-resistant strains of Staphylococcus aureus resistant to quinolones. J. Clin. Microbiol., 27, 335-336.

8. Blumberg, H., Rimland, P., Croll, D. J., Terry, P. and Wachsmuth, I. K. (1991): Rapid development of ciprofloxacin-resistance in methicillinsusceptible and -resistant Staphylococcus aureus. J. Infect. Dis., 163, 12791985.

9. Trucksis, M., Hooper, D. C. and Wolfson, J. S. (1991): Emerging resistance to fluoroquinolones in staphylococci: an alert. Ann. Intern. Med., 114, 424426.

10. Jones, R. N. (1992b): Fluroquinolone resistance: an evolving national problem or just a problem for some physicians? Diagn. Microbiol. Infect. Dis., $15,177-179$. 
11. Sanders, C. C. (1992): Review of preclinical studies of ofloxacin. Clin. Infect. Dis., 14, 526-538.

12. Forstall, G. J., Knapp, C. C. and Washington, J. A. (1991): Activity of new quinolones against ciprofloxacin-resistant staphylococci. Antimicrob. Agents Chemother., 35, 1679-1681.

13. National Committee for Clinical Laboratory Standards (NCCLS) (1993a): Approved standard M2-A5: performance standards for antimicrobial disk susceptibility tests, 5 th ed., NCCLS, Villanova, PA.

14. National Committee for Clinical Laboratory Standards (NCCLS) (1993b): Approved standard M7-A3: Standard methods for dilution antimicrobial susceptibility tests for bacteria which grow aerobically, 3rd ed., NCCLS, Villanova, PA.

15. Jones, N. R., Daryl, H. J. and the North American ofloxacin study group (1994): North American (United States and Canada) comparative susceptibility of two fluoroquinolones: ofloxacin and ciprofloxacin. A 53-medical center sample of spectrum of activity. Diagn. Microbiol. Infect. Dis., 18, 4956 .

16. Jones, N. R., Kehberg, E. N., Erwin, M. E., Anderson, S. C. and Fluoroquinolone resistance surveillance group (1994): Prevalence of important pathogens and antimicrobial activity of parenteral drugs at numerous medical centers in the United States I. Study on the threat of emerging resistances: real or perceived? Diagn. Microbiol. Infect. Dis., 19, 203-215.

17. Hossain, N., Armed, Z. and Mostafi, M. (1996): Urinary tract infection in children. A study of 50 cases. Bangladesh Armed Forces Med. J., vol. XX, 61-64.

18. Jinnah, F., Islam, M. S., Rumi, M. A. K., Morshed, M. G. and Huq, F. (1996): Drug sensitivity pattenr of Escherichia coli causing urinary tract infection in Diabetic and Non-Diabetic patients. J. Int. Med. Res., 24, 296-301.

19. Khan, D. M. and Bhutta, Z. A. (1995): Ciprofloxacin in multiresitasnt infection in childhood: an audit. J. Pak. Med. Assoc., 45, 147-150.

20. Wolfson, J. S. and Hooper, C. D. (1989): Bacterial resistances to quinolones: Mechanisms and clinical importance. Rev. Infect. Dis., 11, S960-S968.

21. Piddock, L. J. V. and Wise, R. (1989): Mechanisms of resistance to quinolones and clinical perspective. J. Antimicrob. Chemother., 23, 475483.

22. Deguchi, T., Yasuda, M., Kawamura, T., Nakano, M., Ozeki, S., Kanematsu, E., Nishino, Y. and Kawada, Y. (1997): Improved antimicrobial activity of DU-6859a, a new fluoroquinolone, against quinolone resistant Klebsiella pneumoniae and Enterobacter cloacae isolates with alterations in GyrA and Par C proteins. Antimicrob. Agents Chemther., 41, 2544-2546. 